\title{
Portable Monitoring / Diagnostic System based on the Combining of Real Time WSN with GSM and Internet
}

\author{
Samir Jasim Mohammed \\ Electrical Engineering Dep. \\ Babylon University \\ Hilla, Iraq
}

\author{
Mahmoud Shaker Nasr \\ Electrical Engineering Dep. \\ Babylon University \\ Hilla, Iraq
}

\author{
Ali Jasim Ramadhan \\ Electrical Engineering Dep. \\ Babylon University \\ Hilla, Iraq
}

\begin{abstract}
The development of the healthcare systems, by combining Ehealth, I-health and GSM-health together, recently became the focus of attention of researchers. In this research, will develop our research that practically designed in the name of "Real Time Portable Health Care PP, GSR, BG, EMG with Alarm System Based on WSN" to assist in the development of the health situation in the hospitals. The system develops by supporting GSM and Internet together in the wireless sensor network (WSN) under ZigBee protocol and by supporting the printing of medical reports. Where the implemented system measures the biomedical data utilizing a group of biomedical sensors, which are patient position sensor (PPS), galvanic skin response sensor (GSR), blood glucose sensor (BGS) and electromyography sensor (EMG).
\end{abstract}

The System monitors the sleep position and skin conductance (indicator to patient's calm) of the patients in real time with alarm for each abnormal case in the base station (BS) and lobbies, and sends SMS alarm to doctor's phone, and it follows-up patient's glucose rate and diagnosis the patient's electromyography status. Moreover, it sends the glucose data to doctor's phone and uploads it to the special web site by using the GSM and Internet technologies. In addition, it introduces a printed hard copy medical report. Moreover, the system stores the patient information and his health data in order to use it for any archiving process or statistical study.

In the achievement system, three nodes are considered according to the facilities available but it stays monitor / diagnosis for an open node number. The system is implemented practically and applied for some persons under supervision of the specialist physicians, and the results obtained are very satisfied and present a great service to human care. All obtained results are presented in this paper.

\section{Keywords}

E-health, I-health, GSM-health, WSN, ZigBee, PPS, GSR, BGS, EMG.

\section{INTRODUCTION}

A monitoring / diagnosing system uses wireless technology to send physiological data from the patient's body to the BS in real time. Where, the patients can wear sensing devices that sense physiological conditions and send it to their doctors in real time and can be used GSM and Internet to allow the doctors monitoring / diagnosing the patients' status remotely.

Due to their flexibility, low power and low cost characteristics, the WSNs are used in health care [1]. By using the wireless sensor network (WSN) technology, can deal with group of biomedical sensors for each one of group of patients [2]. The wireless sensor networks are consisted by small electronic devices called nodes, and the functions of them to sense, transmit and receive a specific signal that is picked up by the specific sensor that is selected by depending on the sensing environment [3]. The WSN connects all these nodes and it may contain 1000's of nodes. Such systems can make changes in the way we live and work in the near future [4]. By combining WSN and GSM technologies, they will provide valuable real time information enabling the doctors to monitor and analyze a patient's current and previous state of health [5]. Today, the combining of WSN and Internet technologies can integrate healthcare systems to add more important services such as diagnosis and monitoring in remote area [6].

In the presented paper, introduces the designed portable real time wireless medical system for monitoring / diagnosing the patients' status based on E-health / GSM-health / I-health and WSN under ZigBee protocol. Where, it is implemented practically and applied for some persons under supervision of the specialist medical physician, and the results obtained are very satisfied and present a great service to human care.

\section{OVERALL SYSTEM DESCRIPTION}

The main goal of the system is for monitoring / diagnostic patient's sleep position, skin conductance, blood glucose and electromyography based on real time ZigBee-WSN, GSM, Internet, alarming and printing report subsystems. Where it has ability to send SMS alarm in the abnormal case of sleep position and skin conductance, and sends notification about blood glucose to doctors' phone by using GSM technology, and the doctor can follow-up the patient's blood glucose rate remotely by using Internet technology. The proposed system based on wireless sensor network (WSN) that collect the sensing data from the patients nodes and send it wirelessly to monitoring / diagnostic system in the base station (BS), which enables the person responsible (doctor) to monitor / diagnostic the patients remotely.

In the presented system, three nodes (lobbies) are considered according to the facilities available but it stays monitor / diagnosis for unlimited patient numbers. The overall proposed system. Where, the main parts of the proposed system are Sensing \& Data Transceiver, Base Station (BS), GSM and Internet Subsystems (see Figure 1). 


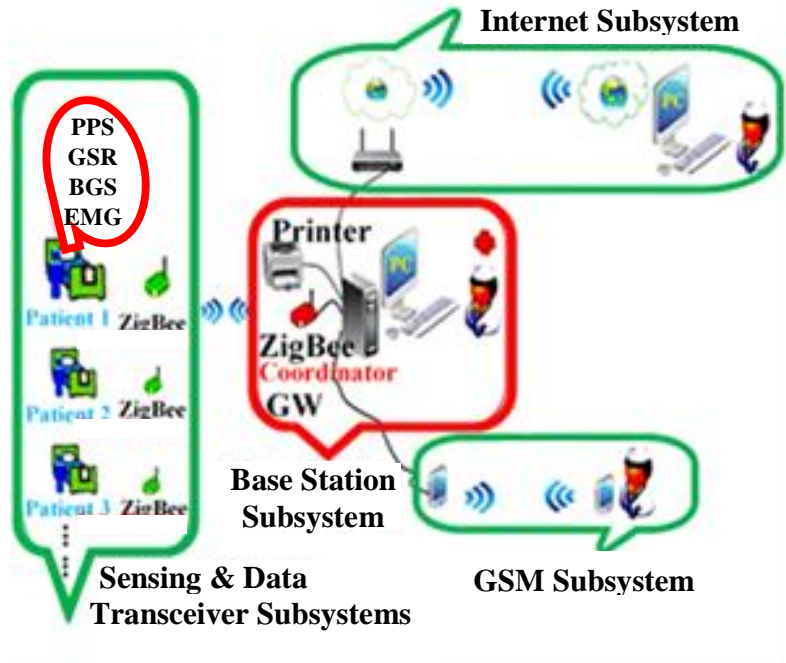

Fig. 1. The overall proposed system

\subsection{Sensing \& Data Transceiver Subsystems}

Each patient is represented by a node, which consists of the required sensors, processor, transceiver and power supply.

\subsubsection{Biomedical Sensors Subsystem}

The patient node has four sensors, which are patient position sensor (PPS), galvanic skin response sensor (GSR), blood glucose sensor (BGS) and electromyography sensor (EMG). The measurement process for these sensors is explained in following:

\section{Patient Position Measurement}

A position sensor is a device that measures the position. It can be either an absolute position sensor or a relative one accelerometer or displacement [7]. Where the accelerometer converts the acceleration from motion or gravity to either analog or digital signals [8]. Digital, capacitive, $3.3 \mathrm{v}, \pm 2 \mathrm{~g}$ and 3 -axis accelerometer is selected as sensor sleep position. The offset axis is $(3.3 \mathrm{v} / 2 \mathrm{~g})=1.65$, where if an axis voltage is above 1.65 it considered positive and other case negative. The acceleration is packed with DSP function that detect the five sleep positions. The blocks diagram of it are shown in Figure (2). Where the voltage of each axis is calculated and compared between them to calculate the larger axis voltage that assumed as $1 \mathrm{~g}$ in DSP function while the others are $0 \mathrm{~g}$.

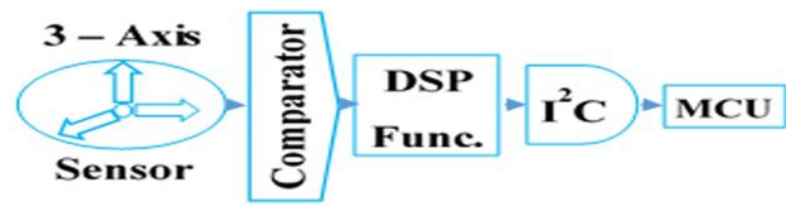

Fig. 2. Accelerometer blocks diagram

The sensing axis will send to the MCU (Pin A4) through $\mathrm{I}^{2} \mathrm{C}$ and the MCU programmed to display the real position (see Table 1). The method for setting the position sensor is shown in Figure (3).

Table 1 Detection of Sleep Positions

\begin{tabular}{|c|c|c|c|c|c|}
\hline X, Y, Z & $\mathbf{0 , 1 , 0}$ & $-1,0,0$ & $1,0,0$ & $0,-1,0$ & $0,0,1$ \\
\hline DSP & 1 & 2 & 3 & 4 & 5 \\
\hline MCU & Supine & Left & Right & Prone & Stand-Sit \\
\hline
\end{tabular}

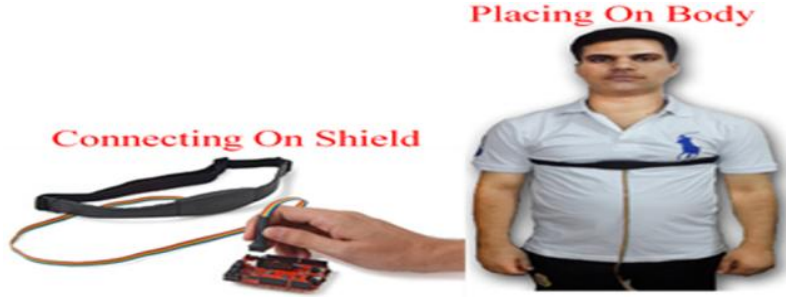

Fig. 3. Setup of position sensor

\section{Galvanic Skin Response Measurement}

The GSR is one of many electro dermal responses that is a medical term for changes in skin conductance due to a psychological condition [9]. The normal skin conductance is between $2 \mu$ Siemens to $10 \mu$ Siemens [10]. The GSR provides a measure of skin conductance by attaching two electrodes on the top of the index and middle fingers and the blocks diagram of its measurement are shown in Figure (4). Because of the voltage resulting is high impedance, it is important to buffer the signal by using the two op-Amp MCP6002. Then, it is passed through the difference Amp. TI INA114 to calculate the difference electrode voltage and then filtered to remove any high frequency noise, where the GSR is a low frequency $(1-2 \mathrm{~Hz})$ signal. The output signal will feed to the MCU through the pin (A2) and the MCU will read it and calculate the skin conductance value.

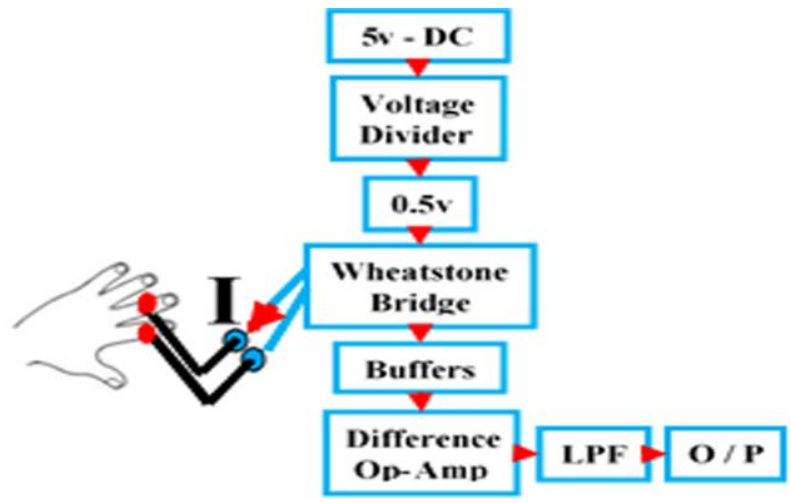

Fig. 4. GSR blocks diagram

The method of setting the GSR sensor is shown in Figure (5).

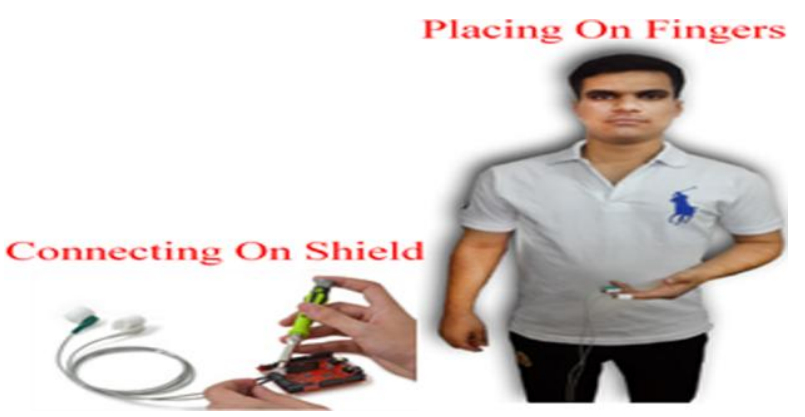

Fig. 5. Setup of GSR sensor

\section{Blood Glucose Measurement}

The glucose is a carbohydrate, which is a source of energy for the human body. Insulin and glucagon are hormones released by the pancreas that have opposite effects on human body [11]. The glucose meter in this work is based on electrochemical test strips. Where it is implemented by using PIC16LF178X device and its blocks diagram are shown in 
Figure (6). Where test strip consists of reference electrode and working electrode [12].

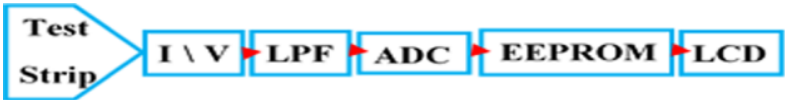

Fig. 6. Blocks diagram of glucose measurement

The measured current will feed to current to voltage conversion and filter from above $8 \mathrm{~Hz}$ signals, and then feed to 12-bit ADC. The glucometer starts capturing glucose voltage at ADC channel, store data in EEPROM and display it on the LCD after less than 7 seconds of placing the blood sample as shown in Figure (7). The power to glucose meter demo board can be supplied from on board lithium battery (3V CR2032).

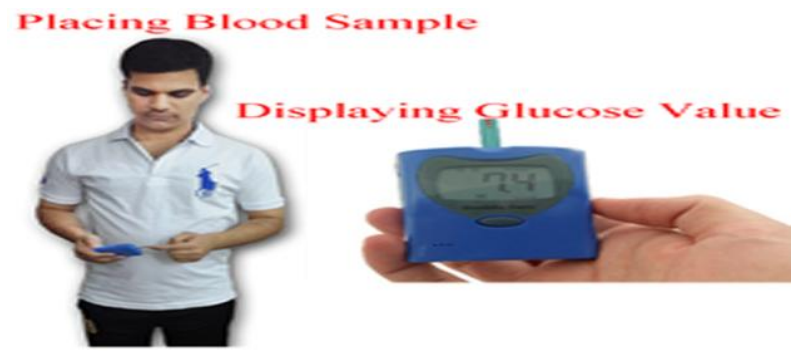

Fig. 7. Measuring blood glucose

\section{Electromyography Measurement}

Electromyography is the process of recording and analyzing myoelectric signals that formed by physiological changes in muscle fiber membranes [13]. The blocks diagram of EMG measurement are shown in Figure (8). A surface, gelled, passive electrode and difference op-amp. AD8221 are used for picking up the EMG signals. The band-pass filter (BPF) of 10 to $500 \mathrm{~Hz}$ is used to remove the heartbeats. After rectifying (HWR) and integrating the signal, it will feed to the MCU (pin A0) to calculate the EMG values.

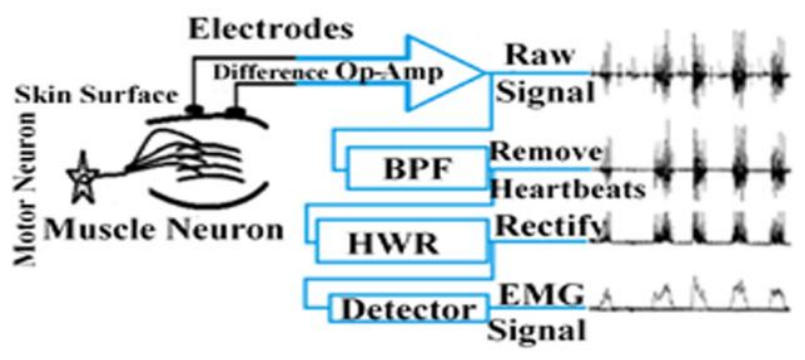

Fig. 8. Blocks diagram of EMG measurement

Where the signal can range from 0 to $1.5 \mathrm{mV}$ (RMS) and from 30 to $300 \mathrm{~Hz}$ [14]. The method for setting the EMG sensor is shown in Figure (9).

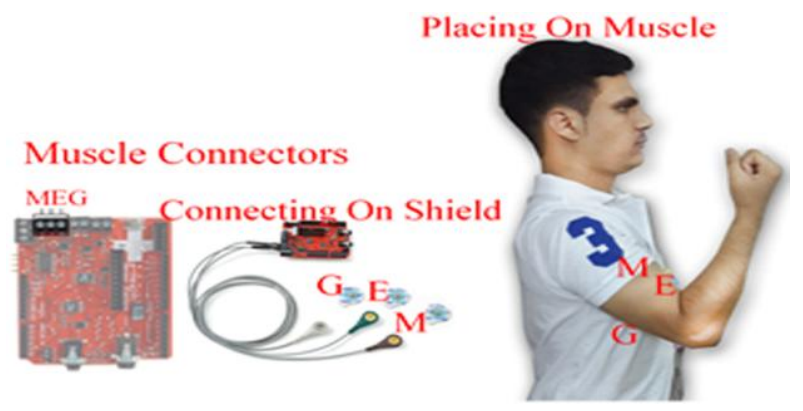

Fig. 9. Setup of EMG sensor

\subsubsection{Processor (MCU) Subsystem}

The MCU ATmega328 is used in this work, and the Arduino platform is selected which contains ATmega328 that is open source and easy to use hardware and software.

\subsubsection{Transceiver Subsystem}

In this work, XBee Series 2 module transceiver is used to connect the patients nodes with BS, where it configured by using X-CTU program. These XBees (in patients nodes) configured as End-Devices firmware by using AT command mode. Because of the ZigBee protocol is supported star, cluster tree and mesh network topologies, so selected star topology because it is easy to synchronize, low power and very low delay.

\subsubsection{Power Subsystem}

A rechargeable battery is used in the each patient node. Therefore, there is a limit time for node operation, and to solve that, the MCU is power $\mathrm{ON}$ each sensor that responds from BS to send its data and then power off it, and used the (SLEEP_MODE_IDLE) that disable the unnecessary functions of the MCU. The power consumption of the main hardware components that used in this system are measured (see Table 2).

Table 2 Power Consumption of Active and Sleep modes

\begin{tabular}{|c|c|c|c|c|c|c|}
\hline Comp. & MCU & PPS & GSR & BGS & EMG & XBee \\
\hline Active & $15 \mathrm{~mA}$ & $\begin{array}{c}165 \mu \\
\mathrm{A}\end{array}$ & $\mathbf{0 . 5 m A}$ & $\begin{array}{c}\text { 0A } \\
\text { External }\end{array}$ & $0.7 \mathrm{~mA}$ & $40 \mathrm{~mA}$ \\
\hline Sleep & $\mathbf{0 . 6 m A}$ & $\mathbf{0 A}$ & $\mathbf{0 A}$ & $\begin{array}{c}\text { 0A } \\
\text { External }\end{array}$ & $\mathbf{0 A}$ & $10 \mu \mathrm{A}$ \\
\hline
\end{tabular}

\subsection{Base Station (BS) Subsystem}

The base station consists of the Gateway (GW), Computer (PC) and Printing subsystems.

\subsubsection{Gateway $(G W)$ Subsystem}

The GW goal is for sending or receiving data to or from the patients' nodes and passing it to or from the PC in BS. It consists of two components, which are XBee and its USB explorer. The XBee in GW is programmed as a coordinator. In this system, the MCU is programmed as five cases structures to perform five operations in each node. Therefore, the GW sends a special case number to the MCU to perform the specified operation.

\subsubsection{Computer (PC) Subsystem}

The process of monitoring, analyzing and recording data are done on the PC. In the implemented system, used LabVIEW software (SW) to do these processing, because it has a graphical user interface (GUI) window can handle data quickly and easily interface with external hardware. The SW uses serial connection as input and output data, which interface with the XBee serial connections of the GW. In the implemented SW, there are three operations implemented on five GUI windows (see Table 3).

Table 3 GUI Windows Classification

\begin{tabular}{|c|c|c|c|c|c|}
\hline Wind. & $\begin{array}{c}\text { Nodes } \\
\text { Status }\end{array}$ & $\begin{array}{c}\text { Insertion } \\
\text { Information }\end{array}$ & $\begin{array}{c}\text { PPS } \\
\& \\
\text { GSR }\end{array}$ & BGS & EMG \\
\hline Oper. & Searching & Setup & \multicolumn{3}{|c|}{ Sensing } \\
\hline
\end{tabular}




\subsubsection{Nodes Status GUI Window Operation}

This operation detects the node status and performs the searching for all nodes in the network and stores the active nodes in a string for the usage by other operations.

\subsubsection{Insertion \& Update Patient's Information GUI Window Operation}

This operation is used to set up the main patient information that's needed, which are doctor name, patient's name, patient's gender, patient's age, patient's entry date and doctor's phone. In the proposed system, the patient information is stored as a Text file without needing to send it to the MCU and stored in it, so reduce the system complexity, time delay, increase the security of the personal data and eliminate of the case of loss it when the node's battery be empty.

\subsubsection{Sensing GUI Windows Operation}

There are three GUI windows implemented to sense patient's status and it classified into three operations, which are monitoring sleep position and skin conductance, following up blood glucose rate and diagnosing electromyography status. These operations are the major operation that supported by searching and set up operations.

\subsubsection{Printing Subsystem}

Many cases are needed to provide the patient by medical report about his health status. Therefore, in addition to analysis and display sensing data in a GUI window, added ability to print medical reports by using VISUAL BASIC (VB) software. In the proposed system, report about the patient's blood glucose levels and electromyography status is provided to the patient. The implemented program is saved as exe. File and recall it from the SW. In the following, explaining to the operation of printing report in BGS and EMG GUI windows respectively. The Figure 10 shows the main process of printing report in "BGS" window.

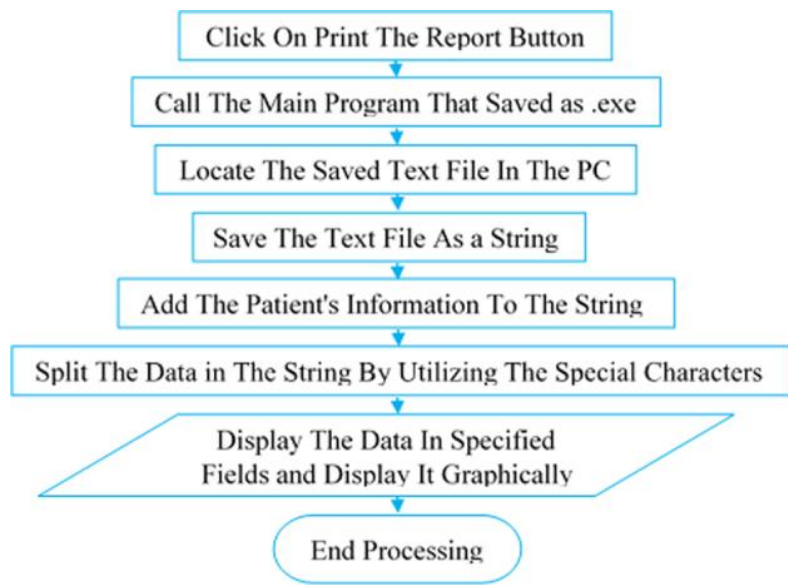

Fig. 10. BGS Printing Report Process

To understand how the print BGS report operation is done, each process in Figure 10 will discuss in detail as follows:

- Click on "Print The Report" button.

- Call the main program that saved as .exe file.

- Locate saved data Text file that stored in PC at BGS operation to read it.

- Save the data as a string.
- Locate the saved information Text file that stored in PC at Setup operation to read it and add it to the string data above.

- Splitting the data in the string by utilizing the special characters.

- Display the data in the specified field and display it graphically, and then print it.

The Figure 11 shows the main process of printing the report in "EMG" window.

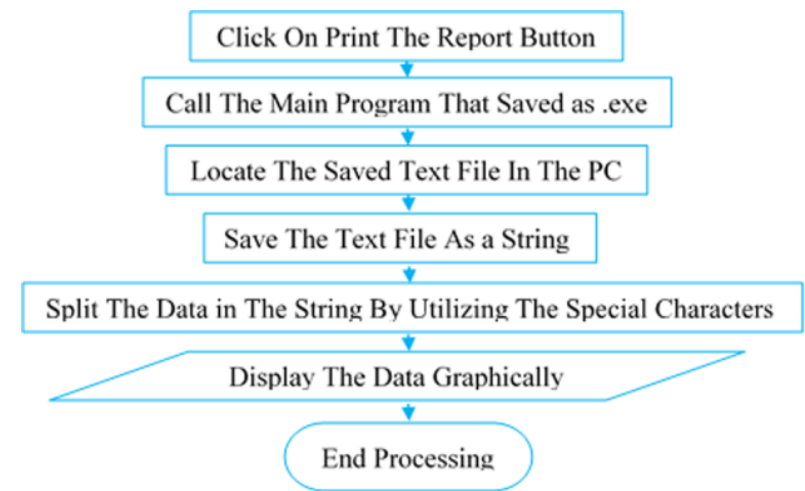

Fig. 11. EMG Printing Report Process

To understand how the print EMG report operation is done, each process in Figure 11 will discuss in detail as follows:

- Click on "Print The Report" button.

- Call the main program that saved as .exe file.

- Locate saved data Text file that stored in PC at EMG operation to read it.

- $\quad$ Save the data as a string.

- Splitting the data in the string by utilizing the special characters

- Display the data graphically, and then print it.

\subsection{GSM Subsystem}

The GSM subsystem is used for remotely following-up by sending SMS alarm for any abnormal case of patient's sleep position and skin conductance, also sends glucose data to doctor's phone, these services are performed by using personal phone device only without using any additional GSM modem or other apparatus, so this method reduces cost, complexity and delay time of the system that's done by interfacing phone with PC directly via USB and messages sending procedure executed by using SMSs AT commands, so doctors be enable to communicate with BS by using SMS or voice calling to give fast and direct necessary guidance. The process of GSM system implementation in BGS window is shown in Figure 12. To understand how the GSM operation is done in (PPS \& GSR) window and in (BGS) window, firstly, each process in Figure 12 will explain in detail as following, and then (PPS \& GSR) implementation process will discussed.

- Click on "Send Notification To Doctor Mobile" button in the BGS GUI window and the Labview executes automatically the following steps.

- $\quad$ Open the mobile phone device serial port.

- $\quad$ Send "AT" command to attend the mobile phone to a new process. 
- Send "AT+CMGF=1" command to attend the mobile phone that the message format is a Text.

- Send "AT+CMGS" command to attend the mobile phone about the selected doctor's phone number.

- Send "Message Data" by recalling the last and previous glucose readings from the string that these two readings displayed on it in the BGS GUI window and they are considered the message data.

- $\quad$ Send "1A" command to end the process, [CTRL+Z] is equivalent to $[1 \mathrm{~A}]$ in ASCII code.

- $\quad$ Close the mobile phone device serial port.

\section{Click On Send Notification To Doctor Mobile Button}

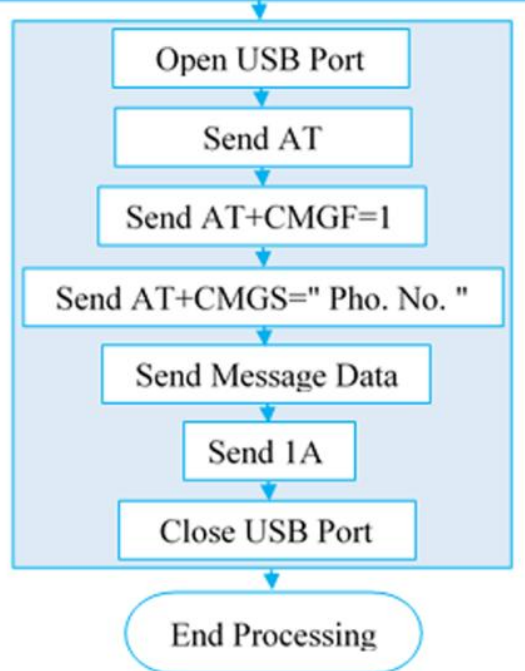

Fig. 12. Process Of Implemented GSM / BGS System

There are only two differences in the implementation of the GSM between the (GSM / BGS) and (GSM / PPS \& GSR), which are:

- In BGS, the process done manually, while in PPS/GSR done automatically, that means there is no "button" to start the process in PPS/GSR.

- In BGS, data is the last and previous glucose readings, while in PPS/GSR the data are the position or the skin conductance.

\subsection{Internet Subsystem}

The Internet subsystem is another excellent service for the presented system. By which remotely following-up glucose levels by uploading glucose data to a specific web site. It is performed by designing a web site that programmed using VB.NET and ASP.NET languages for tasks and GUI of web site respectively, of course, user login feature is added to protect the privacy of patients and feedback comments feature between doctors and patients to take important guidance. The implemented web site done by programming two programs, the first program (Upload Program) combines the measured data with the patient's information in one Text file and upload it to the website link and the second program (Download Program) load the data Text file and publishing it in the web site GUI, and they are saved as exe. file and recall it from the GUI SW. The download program consists of two pages, which are slave and master. The slave page is receiving and storing Text file that is uploaded by the first program from
BS. The master page displays all data depending on the stored files that done in the slave page. The process of Internet implementing is shown in Figure 13.

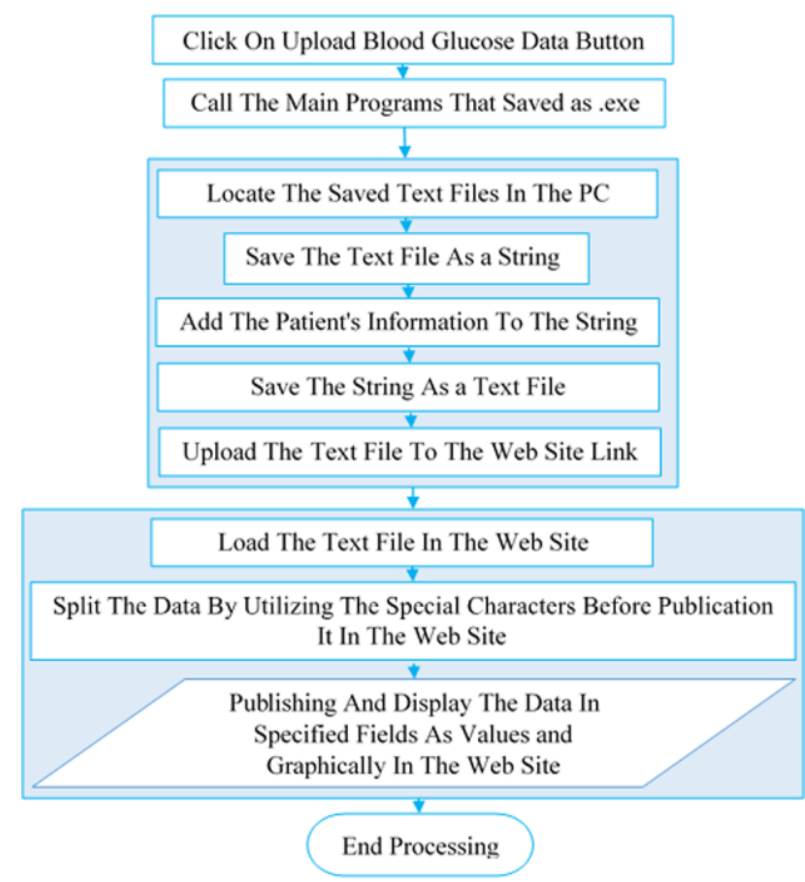

Fig. 13. Process of Internet System Implementation

To understand how this operation is done, each process in Figure 13 will discuss in following:

- Click on "Upload Blood Glucose Data" button.

- Call the main programs that saved as .exe files.

- [Upload Program] Run the program that combined the measured data with the patient information as follows:

$\checkmark$ Locate the saved data Text file that stored in PC at BGS operation to read it.

$\checkmark$ Save the data as a string.

$\checkmark$ Locate the saved information Text file that stored in $\mathrm{PC}$ at Setup operation to read it and add it to the string data above.

$\checkmark$ Save the string as a Text file in the PC.

$\checkmark$ Upload the Text file to the web site link.

- [Download Program] Run program that publishing data on the website as follows:

$\checkmark \quad$ [Slave Page] Download the Text file from the PC server and store it in the folder that carry its' lobby no. .

$\checkmark \quad$ [Master Page] Open the specified folder, read the data and splitting the data in it by utilizing the special characters to publish it.

$\checkmark \quad$ Publish and display all data in the specified field as values and graphs.

\section{OVERALL SYSTEM OPERATION}

In case of monitoring PPS \& GSR, the BS performed touring between the lobbies in three seconds for each lobby. If a PPS or GSR alarm case happens, the GUI window should play 
alarm tone (with indicator) to alert the user in the BS and at the same time the concerned MCU node is turned ON the buzzer and the red PPS or GSR LED in the lobby and also send SMS alarm to doctor's phone. In case of diagnosing EMG, the MCU sends 821 samples of EMG values for every 60 seconds and the user can resend the request several times, all that by depending on the specialist doctors that say that the diagnosis of EMG take a long time about 40 to 60 minutes. The overall implemented system is shown in Figure (14).

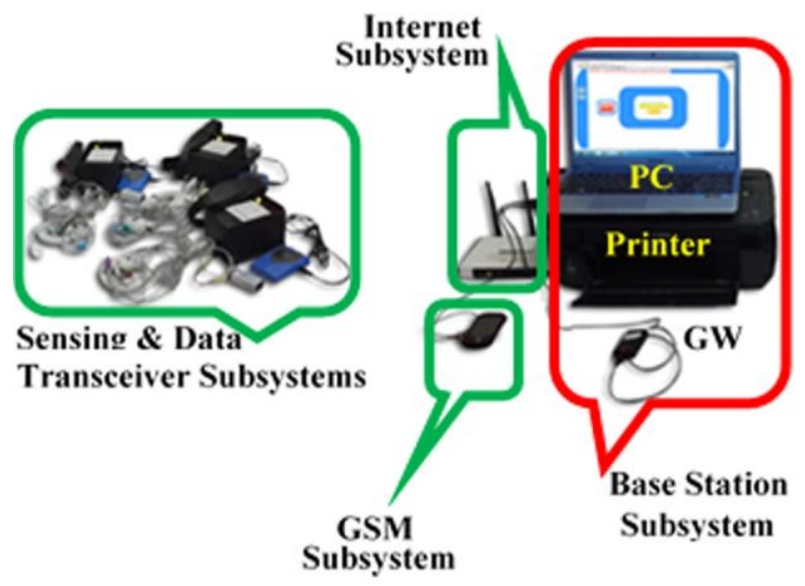

Fig. 14. The Overall Implemented System

\section{SYSTEM FEATURES}

The prototype system has the following features:

- $\quad$ Portable, real time, low power and low cost system.

- Monitoring / diagnosing system and supports alarming, GSM, Internet and printing subsystems.

- Can add other necessary sensors to the system, and the system performs searching and storing process.

\section{RESULTS}

As depicted previously, that the presented system is a prototype and designed for only three patients and the following results are presented according to real cases.

\subsection{Searching Results}

Once the system starts the search operation, the active nodes in the network will display in the specified field as $1,2,3$. If for example node 2 is not active, it displays only nodes 1 and 3 . Figure (15) shows the window of searching process.

Nodes Status | PPS \& GSR | BGS | EMG | Insertion \& Update Patient's Informations

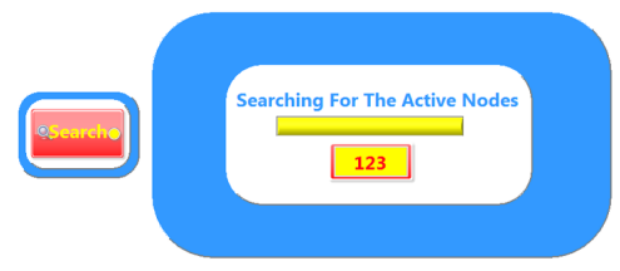

Fig. 15. Searching results

\subsection{Setup Results}

After selecting any patient's lobby, for example no.1, the data are filled as shown in Figure (16), then click on "Save" button.

Nodes Status | PPS \& GSR | BGS | EMG Insertion \& Update Patient's Informations

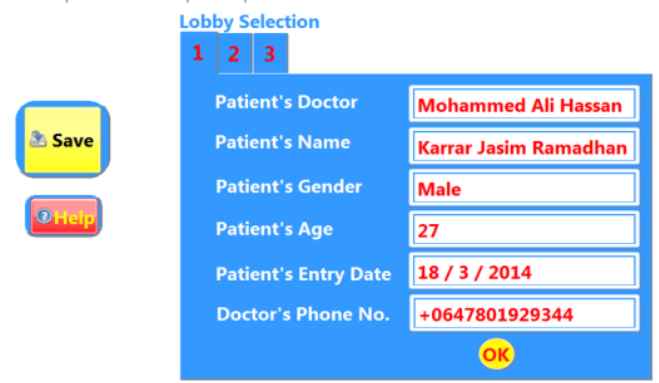

Fig. 16. Setup results

\subsection{Monitoring PPS \& GSR Results}

In PPS alarm setting, the user selects positions that patient should not sleep on there, following settings considered for patient in lobby no.1 (single mode), as example, which are Left and Prone positions.

The results of actual patient position and skin conductance are shown in Figures $(17,18, \& 19)$.

In Figure (17) there is no alarm status, while Figure (18) shows results where patient slept on left position which is not permissible, and Figure (19) shows the results when GSR value is abnormal.

Where SMSs alarm of sleep position and skin conductance that received in the patient's doctor phone are shown in the Figures ( 20 \& 21) respectively.

If the user selected multi-mode monitoring, the system starts to display the results in a sequence.

Of course, the alarm system is applicable for all patient nodes. Nodes Status PPS \& GSR | BGS | EMG | Insertion \& Update Patient's Informations

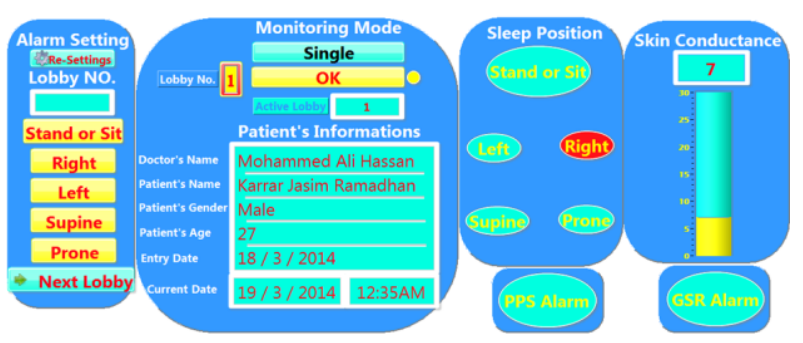

Fig. 17. Monitoring results with no alarm case Nodes Status PPS \& GSR | BGS | EMG | Insertion \& Update Patient's Informations

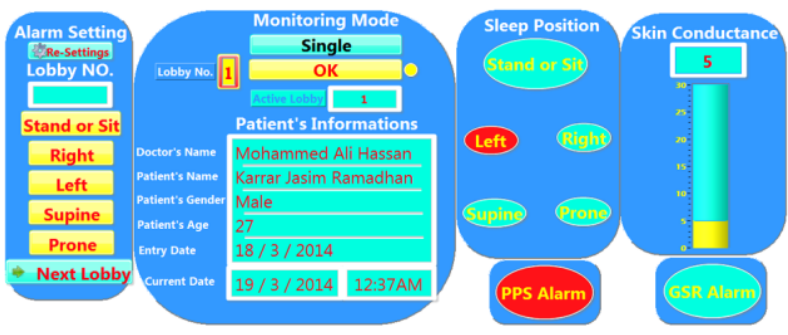

Fig. 18. Monitoring results with PPS alarm case 


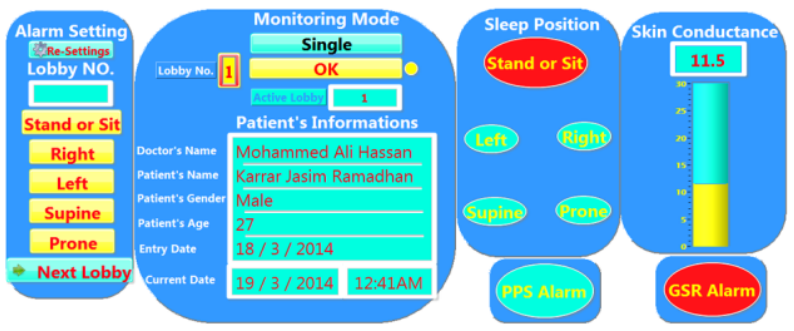

Fig. 19. Monitoring results with GSR alarm case

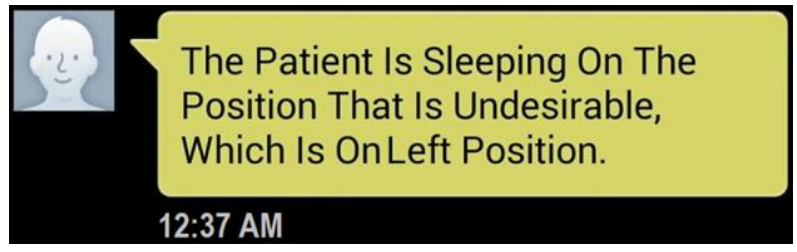

Fig. 20. SMSs Alarm of The Patient's Sleep Position

The Patient Is Not Relax and Has
Skin Conductance Value of 11.5
Micro-Siemens.
12:41 AM

Fig. 21. SMSs Alarm of The Patient's Skin Conductance

\subsection{Following-Up BGS Results}

The user has selected lobby no. 2, as an example, and the results are shown in Figure 22.

The SMS notification of last and previous glucose measured that sent to the doctor's phone is shown in Figure 23.

The patient's information and glucose values are uploaded to the web site, so the doctors can remotely follow-up the patient's status.

As explained previously, the user must login in the website as shown in Figure 24. If the user selected the patient's lobby no.2, as example, the results are displayed in the main website page as shown in Figure 25.

The hard copy report of the patient's blood glucose status is shown in Figure 26.

\begin{tabular}{l|l|l|l} 
Nodes Status & PPS \& GSR BGS & EMG & Insertion \& Update Patient's Informations
\end{tabular}
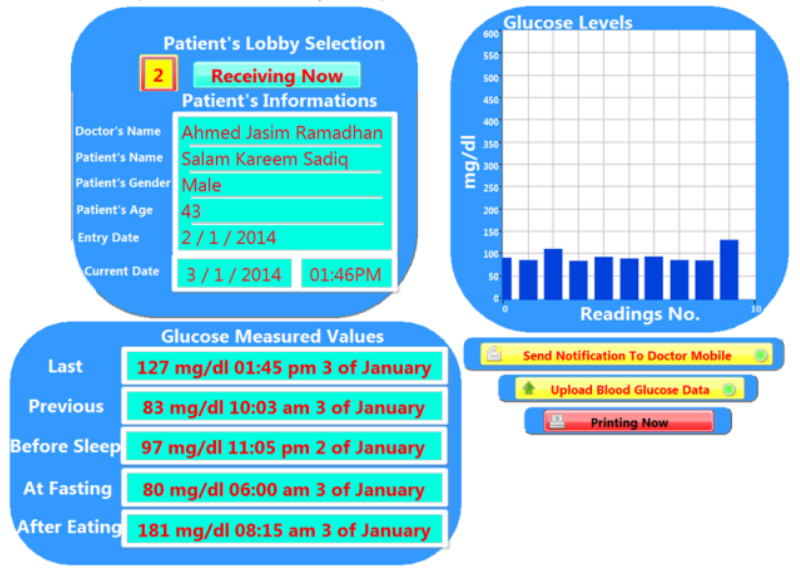

Fig. 22. BGS sensing results

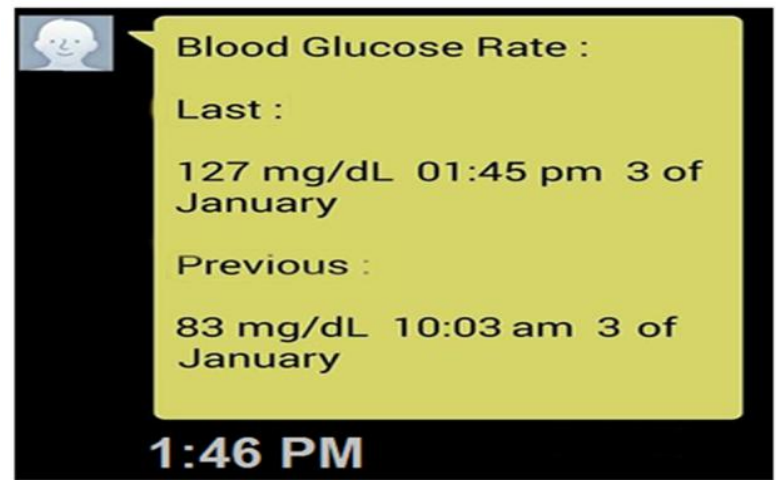

Fig. 23. SMS Notification

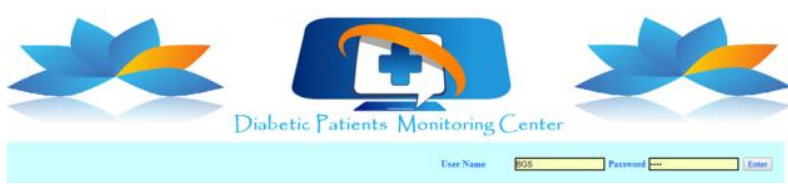

Fig. 24. Web Site - Login Page

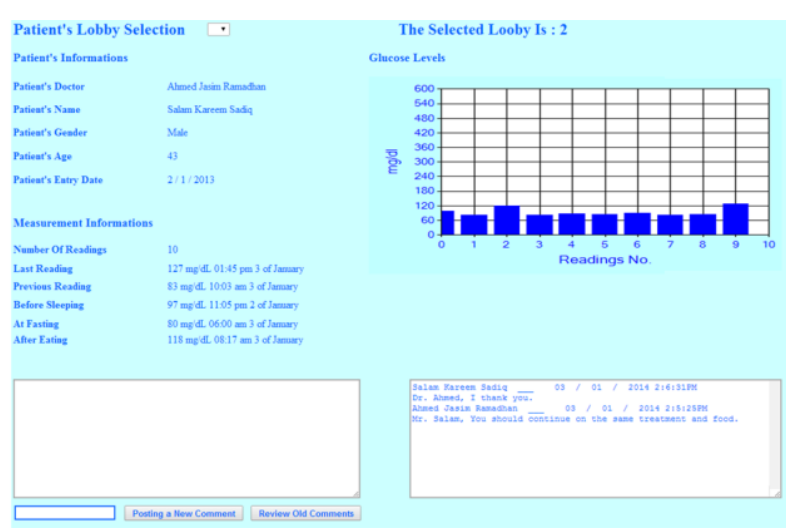

Fig. 25. Web Site - Main Page

Report On The Blood Glucose Rate 03 / $01 / 2014$ 1:47:05PM

Patient's Informations

Patient's Doctor Ahmed Jasim Ramadhan

Patient's Name Salam Kareem Sadiq

Patient's Gender Male

Patient's Age $\quad 43$

Patient's Entry Date 2/1/2013

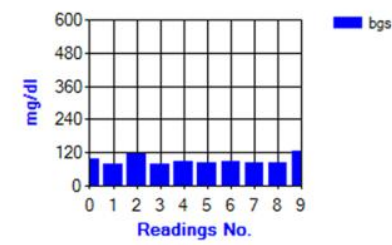

Measurement Informations

No. of Readings 10

Last $\quad 127 \mathrm{mg} / \mathrm{dL} 01: 45 \mathrm{pm} 3$ of January

Previous $\quad 83 \mathrm{mg} / \mathrm{dL} 10: 03$ am 3 of January

Befor Sleeping $\quad 97 \mathrm{mg} / \mathrm{dL} 11: 05 \mathrm{pm} 2$ of January

At Fasting $\quad 80 \mathrm{mg} / \mathrm{dL}$ 06:00 am 3 of January

After Eating $\quad 118 \mathrm{mg} / \mathrm{dL} 08: 15$ am 3 of January

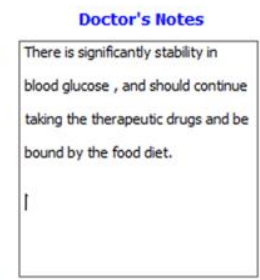

Fig. 26. Glucose Rate Report

\subsection{Diagnosing EMG Results}

The results in the GUI and hard report are shown in Finger 27 and Figure 28 respectively. 


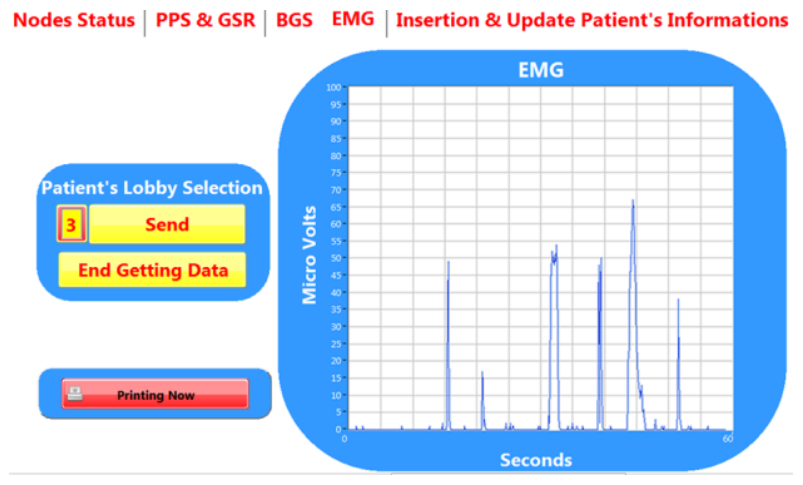

Fig. 27. EMG sensing results
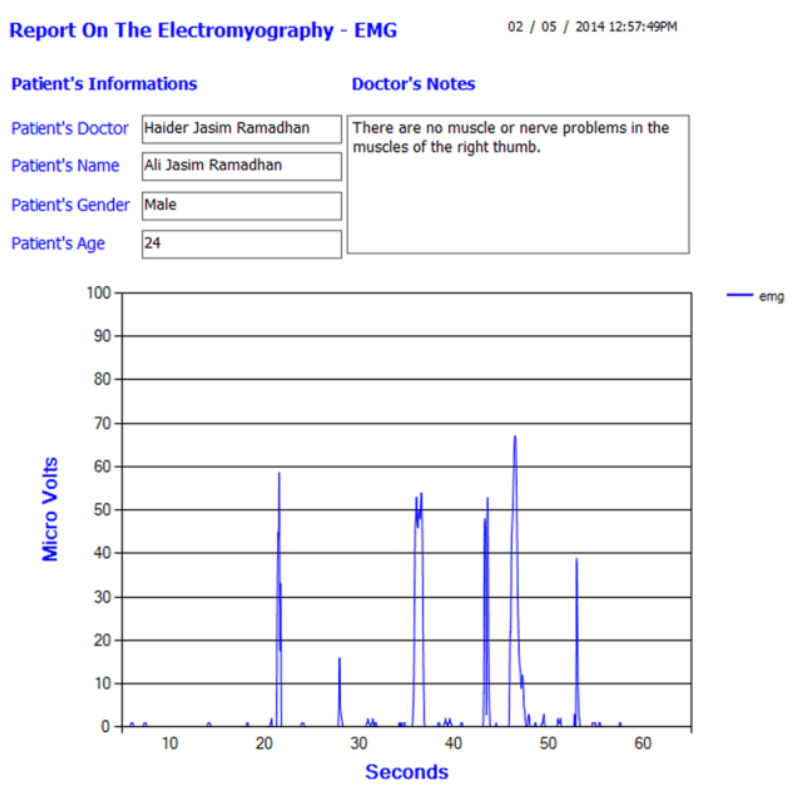

Fig. 28. EMG Diagnostic Report

\section{DISCUSSION}

From the obtained results, the following points can be depicted:

- The process of searching, storing and printing the reports are done successfully.

- The system is working in real time and the SMSs sent to the doctor's phone without mistakes.

- Measured more than 20 samples of the glucose for the same patient and tested the EMG with different period and sample rate without any problems.

- The process of uploading and logging to the website are done without errors.

\section{CONCLUSION AND FUTURE WORK}

During the realization of the presented work, the following notes are depicted as conclusions: the system is designed to support alarm, GSM, Internet and printer together. The system is designed in real time, low power and low cost, and to be portable so used in the hospitals and homes. Can use MATLAB SW, as an example, to study and analysis the stored data. In the future, can improve the system by adding many other sensors and use the implantable glucose sensor to monitor the patient's blood glucose levels continuously. Improve the system by design a database of the patients' health data and information depending on the stored process. Improve the system by design a data history in the designed web site.

\section{ACKNOWLEDGEMENTS}

We offer our thanks to the physicians who were consulted with them in the hospital for their cooperation and advices.

\section{REFERENCES}

[1] Ismail Butun, Salvatore D. Morgera, and Ravi Sankar "A Survey of Intrusion Detection Systems in Wireless Sensor Networks", IEEE, VOL. 16, NO. 1, 2014

[2] Terrance J. Dishongh and Michael McGrath"Wireless Sensor Networks for Healthcare Applications", Intel, ARTECH HOUSE, London, 2010

[3] Jeffrey J. Evans "Undergraduate Research Experiences with Wireless Sensor Networks", IEEE, pp. 7 - 12, 2007

[4] John A. Stankovic, "Wireless Sensor Networks", University of Virginia, USA, 2006

[5] Nitin P. Jain, Preeti N. Jain and Trupti P. Agarkar "An Embedded, GSM based, Multipara meter, Real time Patient Monitoring System and Control - An Implementation for ICU Patients", IEEE, pp. 987-992, 2012

[6] Prabakaran C. R., Vijayakuma R. P. and Suryaprakas H. R. "Web Based Patient Monitoring System Using ARM9 Processor", IJEEDC, Volume- 1, Issue- 7, Sep-2013

[7] Ajay Kumar Bandi "An Integrated Sensor System For Early Fall Detection”, MSc. Thesis, Purdue University, Indiana, 2013

[8] Madhulatha. Kothapu and Ch. Venkat Ratnam "Automatic Control of Robot by Using MEMS Technology", IJPRES, Vol. II/Issue 2 / MAR 2014

[9] G. Shivakumar and P. A. Vijaya "Analysis of Human Emotions Using Galvanic Skin Response and Finger Tip Temperature", IJSE, June 2011

[10] David T. Lykken and Peter H. Venables "Direct Measurement of Skin Conductance: A proposal For Standardization", The Society for Psychophysiological Research, USA, Vol. 8, No.5, 1971

[11] Miriam Garcia Yanez "Glucose Meter Fundamentals and Design“, Freescale Semiconductor, Inc., AN4364, 2013

[12] J. Jirka, M. Prauzek and M. Stankus "Glucose Measuring Device with Advanced Data Processing and Improved Strip Detection“, Elektronika IR Elektrotechnika, 2013

[13] Peter Konrad "The ABC of EMG - A Practical Introduction to Kinesiological Electromyography", Noraxon INC. USA, April 2005

[14] Nikhil Shrirao "Direct Biocontrol of Telemanipulators and VR Environments Using SEMG and Intelligent Systems", MSc. Thesis, University of Akron, USA, 2006 